\title{
Research on feature recognition and optimization of public space in winter city based on the promotion of physical activity A case study of Harbin
}

Shuai LIANG, Harbin Institute of Technology, China; Key Laboratory of Cold Region Urban and Rural Human Settlement Environment Science and Technology, Ministry of Industry and Information Technology, Harbin, 150001, China

Hong LENG, Harbin Institute of Technology, China; Key Laboratory of Cold Region Urban and Rural Human Settlement Environment Science and Technology, Ministry of Industry and Information Technology, Harbin, 150001, China

\begin{abstract}
Urban public space is an important space carrier for residents' physical activity. In winter city, residents' physical activities may present different patterns. With the development of digital media, collecting large-scale and long-term data related to human behavior became easier. Taking Harbin as an example, we collected data from Sina Weibo, and sorted out useful related information to explore users' physical activity types, intensity, and spatial-temporal distribution patterns. The results show that: residents' physical activity show obvious seasonal fluctuations, and the space of individual and group activity preference is significantly different; climate conditions affect the time choice of activities; the type of public spaces has a significant impact on activities. Based on this, a method for optimizing urban public space in cold areas based on physical activity promotion is proposed, which could also provide new insights for promoting the potential health benefits of urban public space in cold climates.
\end{abstract}

\section{Keywords}

Physical activity, cold climate, public space, digital media, optimal design strategy

\section{Introduction}

There have been declines in physical activity in many countries over the past few decades (Brownson et al., 2005; Ng and Popkin, 2012). Lack of physical activity is regarded as the main health challenge facing mankind, which will not only lead to chronic diseases, mental health diseases, decline in quality of life, but also shorten people's life span (US Department of Health and Human Services; Saelens and Handy, 2008). Effective physical activity is an important way to reduce the occurrence of chronic diseases such as obesity and enhance the health of people (Boarnet 2006; Jackson 2003). The British health department has comprehensively summarized the relationship between physical activity and health from the two aspects of prevention and treatment (UK. Department of Health), and put forward the evidence level and effectiveness of physical activity on these two health effects. Generally speaking, improving the level of physical activity has a significant effect on the prevention and treatment of chronic diseases.

Given the limited success of individually-based approaches to behaviour change, public health researchers have increasingly used socio-ecological models to further understand determinants of 
physical activity (Sallis et al., 2008). Such conceptual frame works suggest that the built environment is one important level of influence, as it can facilitate or inhibit participation in physical activity (Sallis et al., 2012). Indeed, public open spaces appear to be key built environment settings that provide opportunities for a variety of physical activity behaviours, such as recreational walking and playing sports (Bedimo-Rung et al., 2005; Kaczynski and Henderson, 2007).

However, in cold areas such as the Northeast China, the severe winter climate conditions lead to the decline of physical activity frequency and the number of activity participants. During the long, cold winter, harsh climate factors, including low temperature, shorter daytime, cold wind, icy and snowy weather, greatly affect the use of public spaces. Affected by the cold climate factors, residents' winter activities are more severely tested, and directly affect the use efficiency in winter. Two different climate environments in winter and summer will produce different activity groups, different activity items and patterns. To adapt to the climate, it was suggested to turn the unfavourable climate environment into an available leisure space with cold climate characteristics (PRESSMAN, 1995), which could increase residents' opportunities for winter activities, improve the use efficiency of public spaces in cold areas, and build cold areas into a "winter friendly" public space environment.

By adopting the large-scale data from weibo, this study explored spatial-temporal distribution patterns of users in cold areas, identified key features for different physical activity types and intensity, and analysed their possible influencing factors to inform the planning for urban public space at the theoretical and practical levels, to encourage and promote more urban residents to participate in daily sports activities such as jogging, slow walking and exercise in a good environment, change the existing bad living habits and lifestyles, and achieve the purpose of reducing the incidence of chronic diseases and improve the health level of urban residents.

\section{Methodology}

\subsection{Study site}

Harbin, a typical winter city, located in the northeast of China, is the capital city of Heilongjiang Province. Its administrative area is rather large with latitude spanning $44^{\circ} 04^{\prime}-46^{\circ} 40^{\prime} \mathrm{N}$, and longitude $125^{\circ} 42^{\prime}$ $-130^{\circ} 10^{\prime} \mathrm{E}$, with a total land area of $53,068 \mathrm{~km}^{2}$. The city center sits on the southern bank of the middle Songhua River. A location map of Harbin is shown in Figure 1. Harbin features a monsoon-influenced, humid continental climate. Due to the Siberian high and its location above 45 degrees north latitude, the city is known for its cold weather and long winter. Winters here are dry and freezing cold, with the

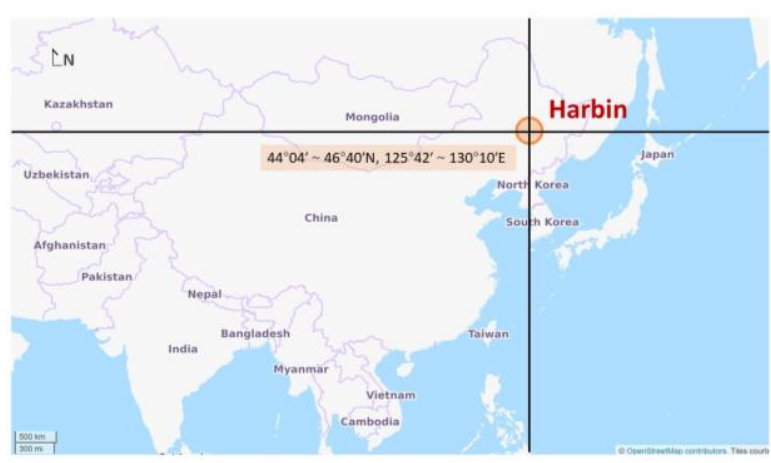
average daily temperature is $-19.7{ }^{\circ} \mathrm{C}$ in winter. Annual low temperatures below $-35.0^{\circ} \mathrm{C}$ are not uncommon. Nicknamed "Ice City" due to its freezingly cold winter, Harbin is decorated by various styles of Ice and snow Sculptures from December to March every year. Spring and autumn constitute brief transition periods with variable wind directions. Summers can be hot, with a July mean temperature of $23.1^{\circ} \mathrm{C}$.

Figure 1. Location of Harbin (Source:Openstreet Map)

\subsection{Data obtaining}

In the process of data mining, the web crawler based on Python is adopted. The python language is concise and the development of crawler program is relatively mature, which can solve this problem. The data is stored in Excel and the photos are stored in local folders, and the numbers are uniformly 
corresponding according to the user ID, which are arranged in ascending order according to the upload time.

This study comprehensively considers the content provided by the open source data of sina Weibo. The final crawling data structure includes: user ID, age, gender, location information, upload time, personal description, customer origin, photos and text comments. The study space covers the check-in points in

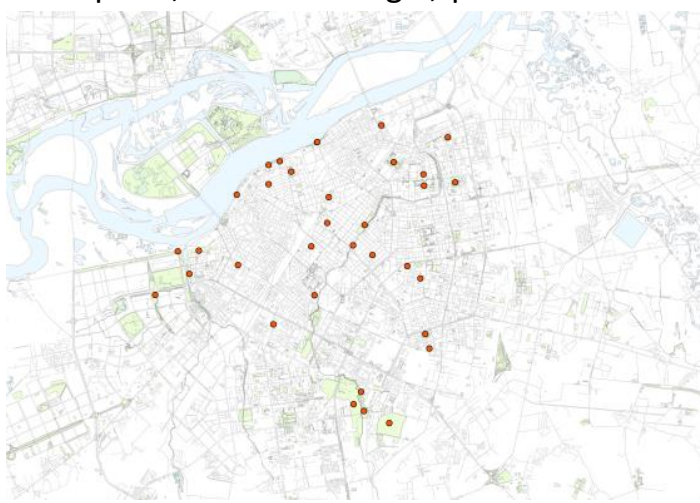
the urban public space in Harbin, and also includes the blue space along the South Bank of Songhua River and Majiagou. After counting the check-in location information of sina Weibo, a total of 31 check-in points located within this range are found (Figure 2). Based on the timeliness of photos and evaluation information, this study tries to cover the data upload volume in spring, summer, autumn and winter, and finds the time interval with the largest sample size. The time span of the final study is from January 2015 to May 2021.

Figure 2. The distribution of samples

\subsection{Data sorting}

We sorted data from the users on Sina Weibo, and got a total of 31348 photos. However, there are many redundant photos that survey useless information about residents physical activity. Therefore, we filtered a total of 13420 effective photos and corresponding behavior activities according to the photo released by the user, including the information related to physical activities. Some of the typical photos are shown below(Figure 3).
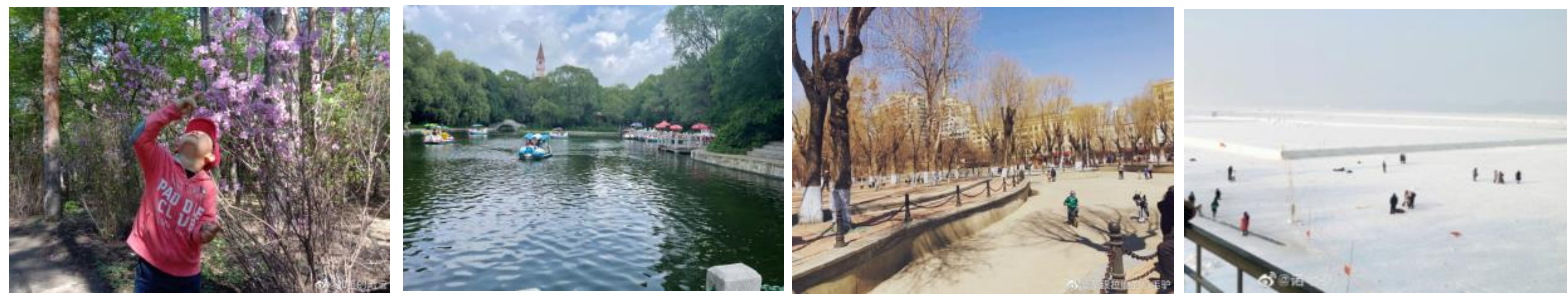

Figure 3. Photos of four seasons from Sina Weibo (Source: Sina weibo)

\section{Physical activity patterns and influencing factors}

\subsection{Basic situation of the users}

By counting the overall microblog entry data, we make a simple statistical summary of the basic gender ratio and age composition of users. We found that the number of female users is a little larger than that of male ones, with about $58 \%$, and $42 \%$, respectively(Figure 4 ). This may also possibly because females are more willing to take photos and share than males. The age of users range from below 10 years old to more than 80 years old. Most of the users are between 30 to 40 years old, followed by $40-50$ years old group. The smallest age group is over 60 years old(Figure 5). The data reflects to a certain extent different genders, age groups of users, based on which a summary of the physical activity patterns and the analysis of influencing factors were obtained. 


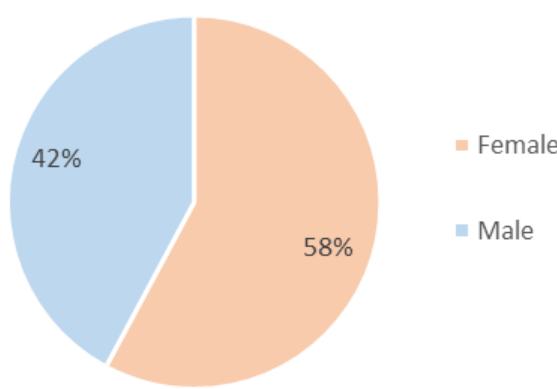

Figure 4. Gender ratio of the users

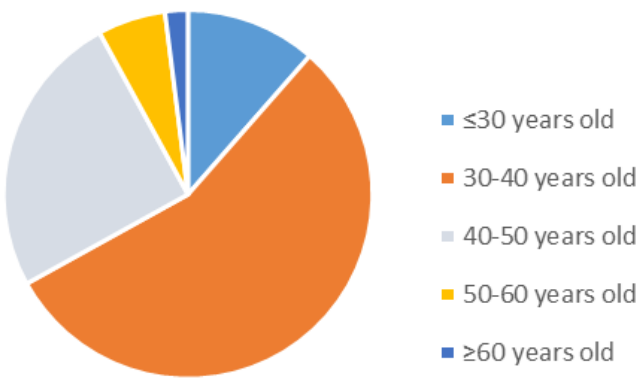

Figure 5. Age group of the users

\subsection{Seasonal variations of users' physical activity patterns}

By identifying information about physical activity from the photos collected, we found that there are significant seasonal variances in the number of activity participants, activity subject, activity intensity and activity type. In terms of the number of activity participants (Figure 6), the number of activities counted in summer was almost as twice as that in winter. In summer, users of all age groups conduct physical activity, among them the elderly account for the largest part. While in winter, the number of juveniles has increased, while the number of old people has decreased significantly. Due to severe cold climate and the slippery path road, less elderly people do physical activity outside, thus the number of activity participants declined sharply.

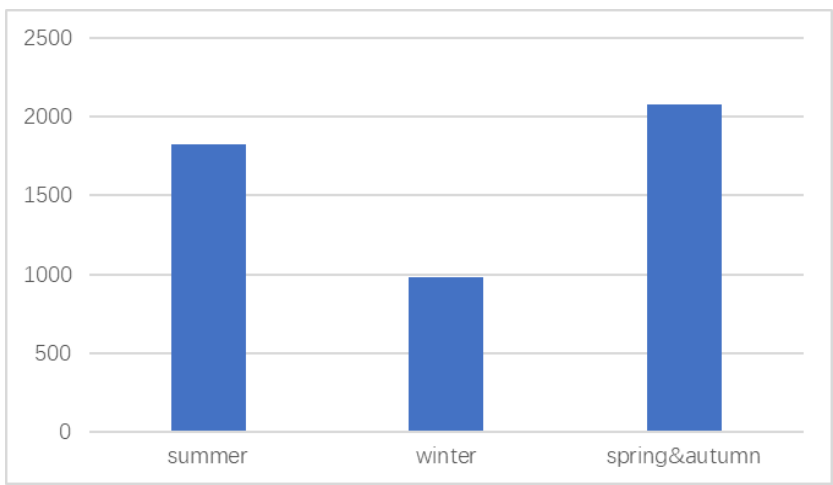

Figure 6. Number of activities of the sample sites in different seasons
There are obvious seasonal differences in activity intensity (Figure 7). In winter, users mainly conduct medium and high-intensity physical activities, and the proportion of lowintensity physical activities is very low. In summer, users mainly focus on low-intensity physical activity, and high-intensity physical activity is significantly lower than that in winter. In spring and autumn seasons, the number of activities of the three intensity levels is close, among which the high-intensity physical activities are relatively low, and the number of the three activity types is basically the same.

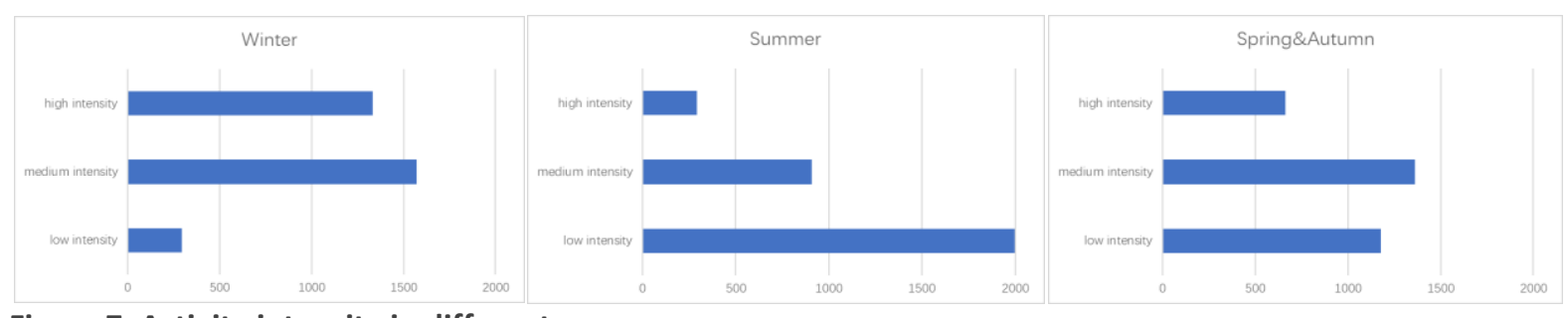

Figure 7. Activity intensity in different seasons 
In terms of activity types, the spring is dominated by sitting and rest, with more walking, more abundant activities in summer, playing in the water, swimming, square dance and other activity types have increased significantly, and there are obviously more fishing activities in autumn. Winter activities are mainly ice and snow sports. The average intensity of physical activity in summer and winter is higher than that in spring and autumn.
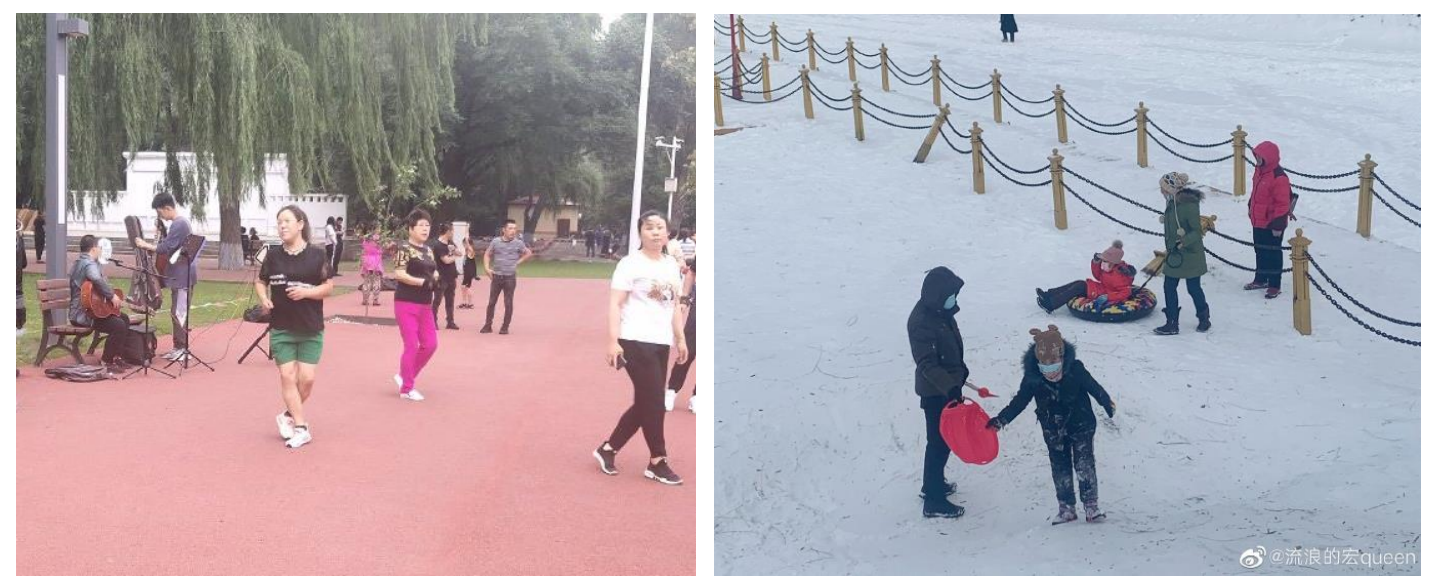

Figure 8. Activity type in different seasons (Source: Sina weibo)

It can be divided into eight main types(Table 1), they are sitting, walking, jogging, sightseeing, accompanying families, playing cards, playing with balls, group activities, ice-snow activities. Some activity types are subject to site restrictions and are enriched by changes in site area and enclosure structure. Some activities are limited by facility support, and the utilization rate of facility support activities is based on the facility around the site. Most of the group activities are located in the area with independent square, convenient transportation and large open space. The middle-aged and elderly people are more likely to sing, dance, chess and cards and other activities. They prefer to reserve a semi private space to watch crowd activities; Ice and water sports are mostly young and middle-aged groups, who like to be close to the natural revetment with perfect infrastructure and beautiful environment; Snowmen and beach climbers are mostly accompanied by teenagers, children and parents. They like to surround a space with a stronger sense of security and a higher safety factor.

Table 1 Activity types identified from weibo users

\begin{tabular}{lllll}
\hline Photos from Weibo & PA Type & \multicolumn{1}{c}{ Elements } & Intensity & Frequency \\
\hline & Sitting & $\begin{array}{l}\text { Sufficient sunshine, mild } \\
\text { wind, comfortable and } \\
\text { sufficient sittings, beautiful } \\
\text { scenes }\end{array}$ & $\square$ Low & $\square$ One time \\
& Walking & $\begin{array}{l}\text { Flat pavement, good vision, } \\
\text { beautiful scenes, enough } \\
\text { space, good lightings }\end{array}$ & $\square$ Short-term \\
& & & $\square$ Ledium & $\square$ Short-term \\
& & & $\square$ High & $\square$ Long-term
\end{tabular}



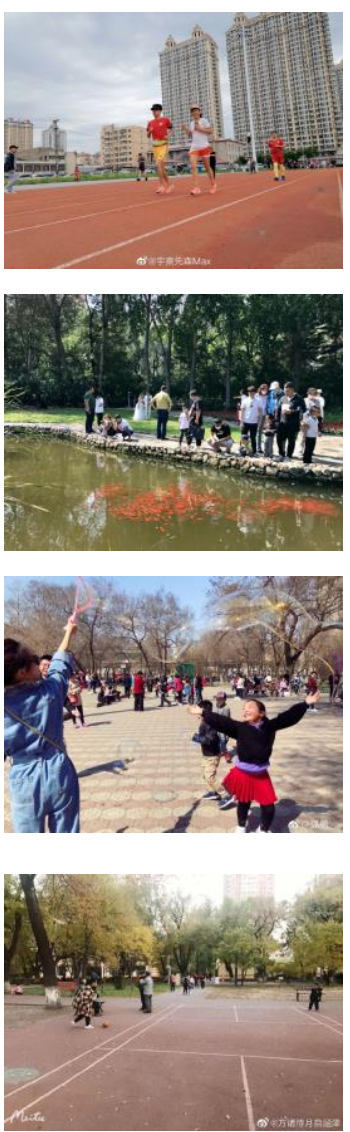

Play

cards

Play

with

balls

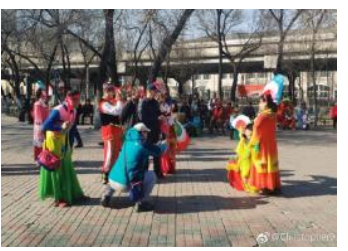

\section{Group} activitie $\mathrm{s}$

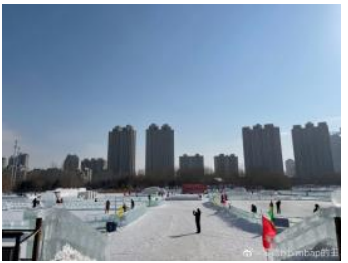

Ice-

snow

activitie

$\mathrm{s}$
Suitable path, flat

pavement, comfortable

environment

Good vision, beautiful

scenes, interesting

sculpture, comfortable

environment

$\square$ Low

$\square$ one time

$\checkmark$ Medium

$\checkmark$ Short-term

$\checkmark$ High $\quad \nabla$ Long-term

$\checkmark$ Low $\quad \nabla$ One time

$\square$ Medium $\square$ Short-term

$\square$ High $\square$ Long-term

$\checkmark$ Low

$\checkmark$ One time

$\checkmark$ Medium

$\square$ Short-term

wind, comfortable

$\checkmark$ High

$\square$ Long-term

Suitable tables, sufficient sunshine, mild wind, warm

$\square$ Low

$\square$ one time

environment, Equipped

with facilities, enough

space, comfortable

environment

$\checkmark$ Medium

$\checkmark$ Short-term

$\checkmark$ High

$\checkmark$ Long-term

VLow

$\square$ one time

Enough space, anti-slip

pavement, sufficient

sunshine, mild wind

$\checkmark$ Medium $\nabla$ Short-term

$\checkmark$ High $\square$ Long-term

Ice and snow appear,

$\checkmark$ Low

$\checkmark$ One time

corresponding equipment,

enough space, rivers and

lakes in winter $\checkmark$ Medium $\nabla$ Short-term

$\checkmark$ High $\quad \square$ Long-term

\subsection{Influencing factors}

Public space is an important place for urban residents to carry out physical activities. It is necessary to further study the problems in detail and analyze the effective urban public space what people need. The demand for good quantity, quality and accessibility public space is welcomed. The lack of humanized elements of urban public space reduces people's daily communication with urban public space, and even makes people ignore the existence of urban daily public activity space. Public space ignores the construction of sports facilities. At present, public space in the city pays more attention to landscape design and functional organization, but lacks detailed design of sports facilities, such as slow track, fitness facilities, and sports facilities that distinguish people, such as sports venues for the elderly, activity venues for children, venues suitable for square dance, etc. especially public spaces at the level of residential areas Space, as the place with the highest daily use rate and closest to life, generally has the 
problems of aging and occupation of facilities in the current city. The lack of enthusiasm to promote residents' movement and the design of public space is an important factor affecting residents' health.

Climate conditions affect the timing of physical activity. Due to the special climatic conditions of the cold city, the number of physical activities and activity types in the four seasons has made a significant difference. In the number of activity participants, there is an obvious peak in summer. The reason is that the microclimate in summer can provide a comfortable environment, which stimulated more outdoor physical activities. The survey shows that although many residents are enthusiastic about winter activities and tend to engage in winter physical exercise in public spaces, there are still few activity venues, imperfect venue facilities, insufficient site radiation area. rarely involves the winter characteristic activities carried by the public space.

Space area or the openness affects the scale of group activities. The difference in spatial form and the sense of enclosure of the space leads to activities of different social scales. Open spaces with large areas and weak enclosures are usually easy to gather on larger scales. Semi-enclosed space with a strong sense of enclosure, which usually attract smaller dynamic group activities, such as snowmen, snowball fights, playing instruments, etc. Encircle closed spaces with a strong sense of synergy are easy to gather smallscale static group activities, such as chess and cards, fitness equipment, sit-ins, picnics, etc.

\section{Implications for urban planning}

How specific public open space features can improve people's awareness of public open space, and in turn how it can improve people's physical activity in relation to public open space should be well examined (Lackey and Kaczynski, 2009). From the perspective of healthy city construction, it is of great significance to consider the influencing factors of physical activity in cold public space in each season, improve the use efficiency of cold public space and promote residents to carry out sports activities, so as to improve residents' physical quality, promote residents' daily communication and build a healthy city.

Cold weather protection: pay attention to the sunshine and wind environment around the fitness place. Due to the influence of winter monsoon and the shortening of sunshine time in cold areas, on the premise of ensuring the sunshine time in fitness places, small structures should be established to strengthen greening, reduce the impact of Winter Monsoon on users' fitness behavior, and increase the time for the elderly in cold areas to stay outdoors in winter.

Expand the size of the site and increase the number of sites. According to the reasonable number of people and the scale of space required for different activities, reasonably develop the unused free sites in the existing stage when the bearing capacity allows. Carry out the reasonable zoning and layout of activities in combination with the actual situation for different users of the site as attributes of the same type of public open space may affect socio-demographic groups differently (Floyd et al., 2008; Kaczynski et al., 2013). Gradually improve the activity category of public space, exploring the specific role of each public open space feature (and subsequent combinations thereof) on physical activity can provide landscape architects with useful information about prioritising features in new and upgraded public open space (Koohsari M.J., 2015), give priority to ensuring the experience of its main activities, gradually improve the different requirements of other activists, and finally increase winter activities such as ice slides according to the needs of activists. We should also maintain the site quality by regularly checking the safety of activity facilities to improve the safety of the site and to make sure that the terrain shall be flat and the slope be reduced, which can ensure the safety of users.

\section{Acknowledgements}

Special thanks are given to Dr. Yang from HIT for the help in getting the data from Sina weibo. 


\section{References}

Brownson, R.C., Boehmer, T.K., Luke, D.A., 2005. Declining rates of physical activity in the United States: what are the contributors? Annu. Rev. Public Health 26, 421-443.

Ng, S.W., Popkin, B.M., 2012. Time use and physical activity: a shift away from movement across the globe. Obes. Rev. 13, 659-680.

U S Department of Health and Human Services. Physical activity and health: a report of the Surgeon General. Atlanta, GA : US Department of Health and Human Services, Centers for Disease Control and Prevention, National Center for Chronic Disease Prevention and Health Promotion, 1996.

Saelens B E, Handy S L. Built Environment Correlates of Walking: A Review[J]. Medicine and Science in Sports and Exercise, 2008, 40(7): 550-566.

Boarnet M G. Planning's Role in Building Healthy Cities: An Introduction to the Special Issue[J]. Journal of the American Planning Association, 2006, 72(1): 5-9.

Jackson L E. The Relationship of Urban Design to Human Health and Condition[J]. Landscape and Urban Planning, 2003, 64(4): 191-200.

U K. Department of Health. At Least Five a Week: Evidence on the Impact of Physical Activity and Its Relationship to Health. A Report from the Chief Medical Officer. 2004.

Sallis, J.F., Owen, N., Fisher, E.B., 2008. Ecological models of health behavior. In: Glanz, K., Rimer, B., Viswanath, K. (Eds.), Health Behavior and Health Education: Theory, Research, and Practice, fourth ed. Jossey-Bass, San Francisco, CA, pp. 465-482.

Sallis, J.F., Floyd, M.F., Rodríguez, D.A., Saelens, B.E., 2012. Role of built environments in physical activity, obesity, and cardiovascular disease. Circulation 125, 729-737.

Bedimo-Rung, A.L., Mowen, A.J., Cohen, D.A., 2005. The significance of parks to physical activity and public health: a conceptual model. Am. J. Prev. Med. 28, 159-168.

Kaczynski, A.T., Henderson, K.A., 2007. Environmental correlates of physical activity: a review of evidence about parks and recreation. Leisure Sci. 29, 315-354.

Yasunaga A, Togo F, Watanabe E, et al. Sex, age, season, and habitual physical activity of older Japanese: the Nakanojo study[J]. Journal of Aging and Physical Activity, 2008, 16(1): 3-13.

PRESSMAN N. Northern cityscape: linking design to climate [M] .Yellowknife: Winter Cities Association, 1995: $3-2$.

"Geographic Location". Basic Facts. Harbin Municipal Government. Archived from the original on 30 January 2013. Retrieved 14 September 2011.

"Chinese ice sculptures melting". BBC. Archived from the original on 5 March 2016. Retrieved 22 April 2014.

Lackey, K.J., Kaczynski, A.T., 2009. Correspondence of perceived vs. Objective proximity to parks and their relationship to park-based physical activity. Int. J. Behav. Nutr. Phys. Act. 6, 53.

Floyd, M.F., Spengler, J.O., Maddock, J.E., Gobster, P.H., Suau, L.J., 2008. Park-based physical activity in diverse communities of two U.S. cities: an observational study. Am. J. Prev. Med. 34, 299-305.

Kaczynski, A.T., Stanis, S.A.W., Besenyi, G.M., Child, S., 2013. Differences in youth and adult physical activity in park settings by sex and race/ethnicity. Preventing Chronic Dis. 10, E42.

Koohsari, M. J. and S. Mavoa, et al. (2015). "Public open space, physical activity, urban design and public health: Concepts, methods and research agenda." Health \& Place 33: 75-82. 